\title{
Le sarcophage de pierre du haut Moyen Âge, des critères pour une étude
}

The early medieval stone sarcophagus, criteria for a study

\section{Fabrice Henrion}

\section{OpenEdition}

\section{Journals}

Édition électronique

URL : https://journals.openedition.org/cel/20584

DOI : $10.4000 /$ cel.20584

ISSN : 2262-208X

\section{Éditeur}

École du Louvre

\section{Référence électronique}

Fabrice Henrion, «Le sarcophage de pierre du haut Moyen Âge, des critères pour une étude », Les Cahiers de l'École du Louvre [En ligne], 17 | 2021, mis en ligne le 26 novembre 2021, consulté le 13 décembre 2021. URL : http://journals.openedition.org/cel/20584 ; DOI : https://doi.org/10.4000/cel. 20584

Ce document a été généré automatiquement le 13 décembre 2021.

\section{cc) (1) $\odot$}

Les Cahiers de l'École du Louvre sont mis à disposition selon les termes de la licence Creative Commons Attribution - Pas d'Utilisation Commerciale - Pas de Modification 4.0 International. 


\section{Le sarcophage de pierre du haut Moyen Âge, des critères pour une étude}

The early medieval stone sarcophagus, criteria for a study

Fabrice Henrion

1 Depuis les années 1950 et l'engagement des travaux du Recueil général des monuments sculptés sous l'impulsion de Jean Hubert, l'étude des sarcophages de pierre a bénéficié d'une attention de plus en plus soutenue et d'une évolution des approches et des méthodes, dans le but, à chaque étape, d'en extraire un maximum d'informations heuristiques. L'époque était encore à considérer surtout les sarcophages ou les fragments décorés, laissant dans l'ombre quantité d'exemplaires qui pourtant participaient entièrement à la connaissance des pratiques et traditions funéraires. Denise Fossart et May Vieillard-Troïekourof ont largement porté à la connaissance de tous l'émergence de nouvelles problématiques, en introduisant la notion de diffusion des productions depuis des ateliers spécifiques et spécialisés. Ce fut une étape importante dans l'étude des sarcophages, même si aujourd'hui on peut aisément remettre en cause l'idée d'ateliers spécialisés dans la sculpture au profit d'une vision, axée sur la caractérisation des mises en œuvre et la reconnaissance des centres de production. Par exemple, on sait maintenant que les sarcophages dits "nivernais", caractérisés par un décor de croix multiples et par la forme du couvercle, étaient produits dans différentes carrières ayant leurs propres aires de diffusion, par le bassin de la Loire ou celui de la Seine. Et il en est de même des sarcophages dits «à bandes de stries gravées alternées ", produits dans l'Auxerrois et diffusés par l'Yonne et la Seine jusqu'à Paris (fig. 1). 
Fig. 1.

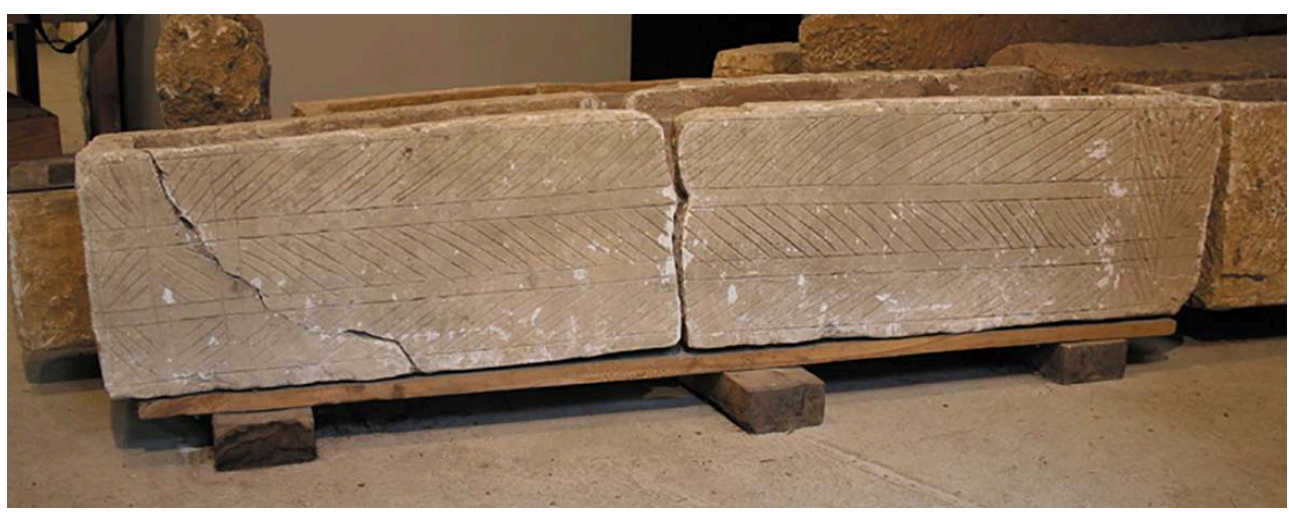

Exemple d'un sarcophage du Groupe A, Escolives-Sainte-Camille (Yonne), S. 005.

(c) F. Henrion

2 Auparavant, Léon Coutil ${ }^{1}$ avait proposé une classification des sarcophages du haut Moyen Âge fondée sur des corpus régionaux, toutefois contrainte par des critères de style inscrits dans un espace géographique ; L. Coutil classe les sarcophages comme il classait les silex taillés. En outre, on pressent dans ce travail sinon une imitation du moins une certaine influence des recherches d'Edmond Leblant sur les sarcophages chrétiens de la Gaule ${ }^{2}$, parfaitement assumée.

Gilbert-Robert Delahaye est longtemps resté un des principaux chercheurs, avec ceux que nous allons évoquer, à travailler sur le sujet et son importante bibliographie, depuis le début des années 1970, témoigne de l'élaboration progressive de typologies, d'abord essentiellement basées sur les décors et la forme des couvercles, puis ouvrant sur l'intérêt de prendre en compte les caractères discriminants, du choix du matériau à l'acte technique de la production. C'est à Jean-François Baratin ${ }^{3}$ que l'on doit d'avoir attiré l'attention sur la nécessité de considérer le matériau, dont l'identification pétrographique ouvre des perspectives sur la reconnaissance des zones potentielles d'extraction (à défaut des carrières elles-mêmes, sauf cas exceptionnels ${ }^{4}$ ), et de là sur les aires et réseaux de diffusion des productions. Jacqueline et Claude Lorenz œuvraient alors dans le même sens et ces chercheurs posaient déjà les bases méthodologiques et d'ouverture des problématiques.

Dans les années 1990 et 2000, fortes de ces expériences, de nouvelles recherches ont pu être engagées en renouvelant les approches et les méthodes, dans une dynamique commune à partir de corpus régionaux, en France mais également en Belgique avec les travaux de Laure-Anne Finoulst ${ }^{5}$. Il convient également de mentionner le Projet Collectif de Recherche Les sarcophages de pierre de la bordure septentrionale du Massif central, sous la direction de Sophie Liegard, dont un des intérêts a été de croiser typologie et pétrographie, confirmant ce qui avait déjà été montré pour la Bourgogne, la production d'un même modèle par plusieurs centres carriers et la confrontation de leurs aires de diffusion.

Ce mouvement, toujours d'actualité, sera ponctué en 2009 par les Journées internationales d'archéologie mérovingienne de Bordeaux consacrées aux sarcophages de l'Antiquité tardive et $\mathrm{du}$ haut Moyen $\hat{A} \mathrm{e}^{6}$, suivies par la publication régulière d'études et de corpus ${ }^{7}$. 
Il est dorénavant acquis que l'on ne peut plus considérer le sarcophage sous un seul de ses aspects, mais bien de prendre en compte l'ensemble des critères constituant son histoire, depuis la carrière jusqu'au lieu d'inhumation.

\section{Rappel des principes méthodologiques}

7 Pour aller au bout de l'étude d'un sarcophage, il est indispensable d'en extraire l'intégralité des informations préservées témoignant de son histoire depuis la carrière jusqu'à la nécropole. Il s'agit donc de localiser le lieu d'extraction et d'en comprendre les techniques, de déterminer la façon dont il a été taillé, façonné, décoré, puis évacué vers un réseau de diffusion avant d'être enfin livré sur le lieu d'utilisation. Il s'agit ainsi de répondre aux questions de provenance en faisant appel à la géologie des matériaux, répondre aux questions de production en déterminant les techniques utilisées à partir de l'étude des traces laissées par les outils, répondre aux questions de formes et de modélisation en mesurant la morphologie des cuves et des couvercles, répondre aux questions d'ordre économique en intégrant les problématiques propres à une réflexion archéologique plus large.

C'est à partir de ces constats et confrontés à la nécessité de disposer d'outils adaptés à la recherche et aux problématiques archéologiques que nous avons été amenés à développer une classification typologique prenant en compte les caractéristiques techniques et morphologiques selon une grille d'analyse et un protocole stricts (fig. 2) ${ }^{8}$.

Fig. 2.

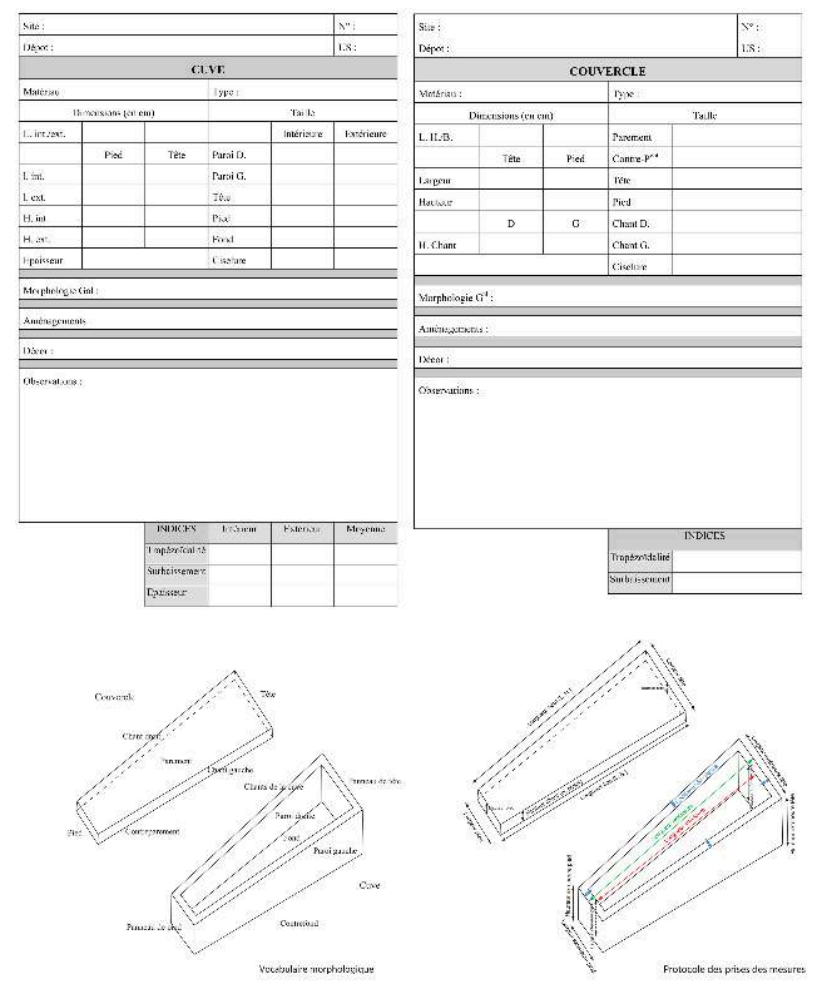

Fiches et protocoles d'enregistrement d'un sarcophage.

(C) F. Henrion, 1998 
Les critères techniques sont déterminés à partir de la reconnaissance des outils utilisés tout au long de la chaîne opératoire, par les traces ou impacts qu'ils auront laissés dans la matière (fig. 3) et, dans de rares cas, par des tracés préparatoires (fig. 4).

Fig. 3.
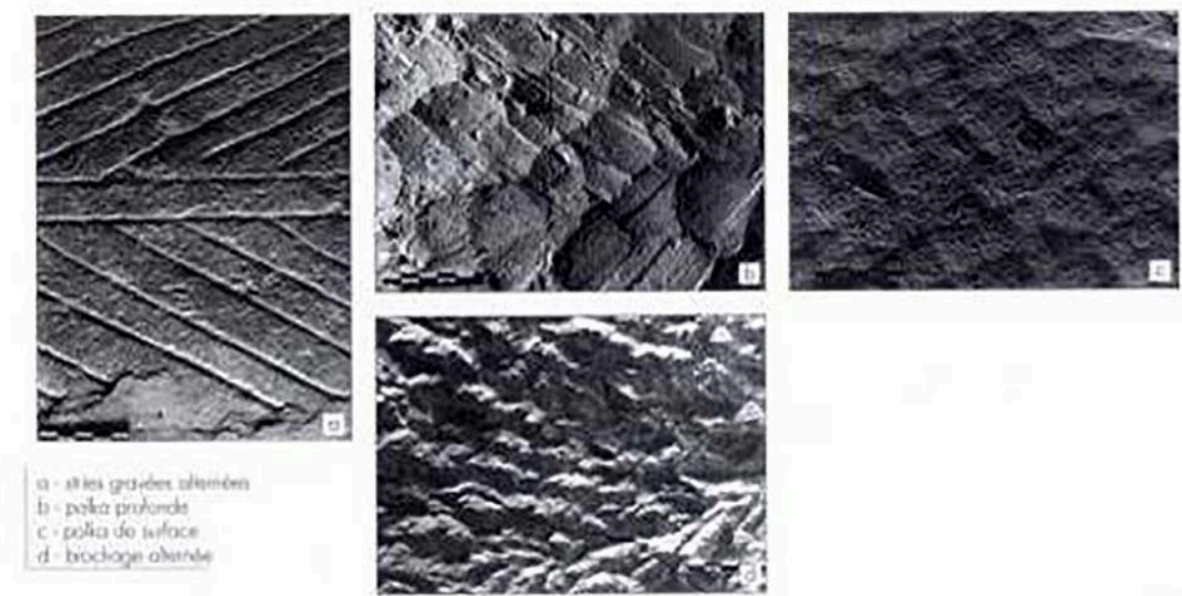

Exemples de traces d'outils utilisés pour la taille d'un sarcophage.

(c) F. Henrion

10 Si l'on peut, dans la plupart des cas, reconnaître l'outil ou les outils, et ainsi inclure l'objet étudié dans un critère technologique, il est indispensable de saisir une donnée difficilement mesurable : la morphologie des cuves et des couvercles.

11 Un système d'indices morphologiques a été mis en place, calculé à partir des dimensions prises sur l'objet : l'indice de trapézoïdalité est le rapport entre la largeur du pied et de la tête (lp / lt), l'indice de surbaissement est le rapport entre la hauteur $\mathrm{du}$ pied et de la tête ( $\mathrm{hp} / \mathrm{ht}$ ) et l'indice d'épaisseur est une moyenne des quatre épaisseurs des parois de la cuve divisée par 10 (moy. / 10).

12 Ces notions de trapézoïdalité, de surbaissement et d'épaisseur restent les mieux adaptées à la perception volumétrique des sarcophages et les indices permettent d'obtenir l'image morphologique stéréotypée de chaque objet étudié.

En outre, on peut dès lors offrir, à partir des indices retenus, un cadre strict à la définition littéraire de la morphologie, de sorte que le discours descriptif soit homogène quel que soit le site étudié :

Fig. 4.

\begin{tabular}{|l|l|l|l|}
\hline Très trapézoïdal & Trapézoïdal & Légèrement trapézoïdal & Rectangulaire \\
\hline 0.00 à 0.35 & 0.36 à 0.60 & 0.61 à 0.95 & 0.96 à 1.00 \\
\hline
\end{tabular}

\begin{tabular}{|l|l|l|l|}
\hline Très surbaissé & Surbaissé & Légèrement surbaissé & Parallèle \\
\hline
\end{tabular}




\begin{tabular}{|l|l|l|l|}
\hline 0.00 à 0.35 & 0.36 à 0.60 & 0.61 à 0.95 & 0.96 à 1.00 \\
\hline
\end{tabular}

\begin{tabular}{|l|l|l|}
\hline Parois minces & Parois moyennes & Parois épaisses \\
\hline 0.00 à 0.50 & 0.51 à 0.75 & 0.76 à $\mathrm{x}$ \\
\hline
\end{tabular}

14 Ainsi, le croisement des données technologiques et morphologiques permet d'extraire les caractères discriminants nécessaires à l'élaboration d'une classification et d'en dégager des familles ou plus précisément des groupes typologiques réunissant les objets présentant les mêmes critères (fig. 5). Une telle classification, en y ajoutant les données pétrographiques, a, entre autres, l'avantage de pouvoir considérer tous les sarcophages de pierre, qu'ils soient entiers ou fragmentaires, en place ou remployés, décorés ou non.

Fig. 5.

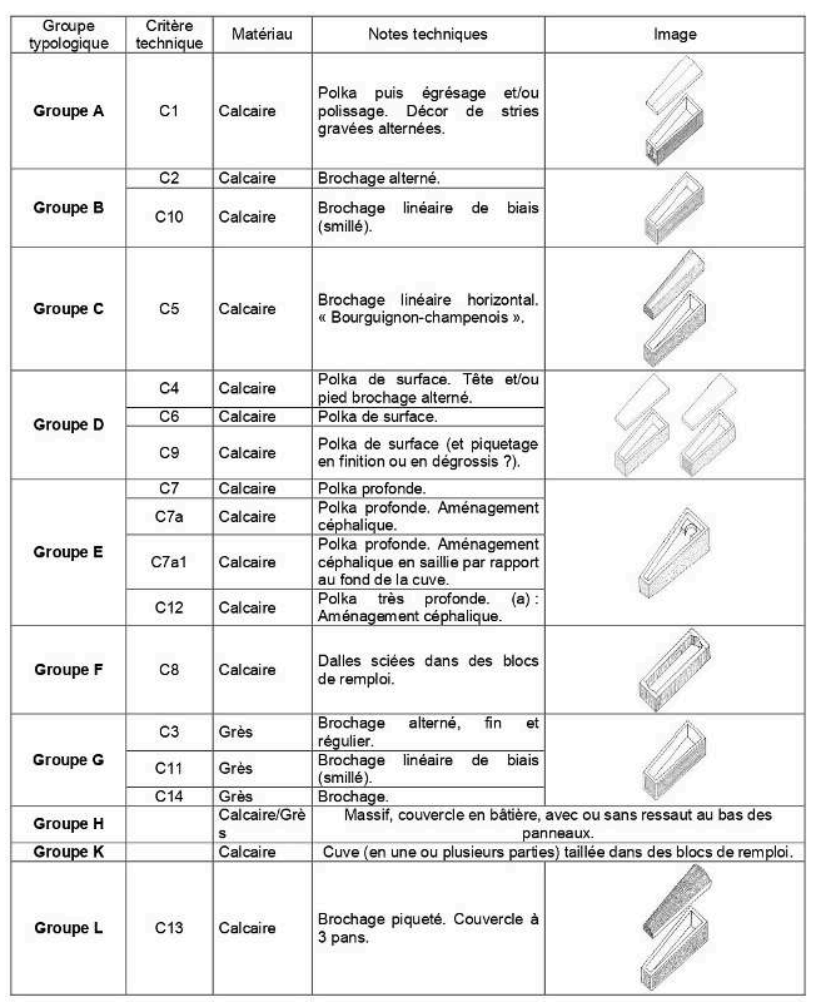

Classification typologique élaborée pour la Bourgogne et la Franche-Comté, puis étendue au quart nord-est de la France.

(c) F. Henrion

\section{Un fragment isolé doit-il être exclu?}

C'est parce que l'archéologue est régulièrement confronté à la présence de fragments de sarcophages, hors contexte ou peu visibles, perturbés par la succession des inhumations ou la reconstruction des espaces bâtis, que la question doit être posée. À l'instar des exemplaires non décorés, les simples fragments, de cuve ou de couvercle, 
ont souvent été ignorés, alors qu'il est possible, dans la plupart des cas, de les raccrocher à une typologie. L'épaisseur du fragment est un critère discriminant, le matériau, ainsi que les traces laissées par l'outil ou les outils employés pour la taille : pic ou broche, polka. Plusieurs outils peuvent être utilisés pour la taille d'un même sarcophage, entre l'évidement de la cuve (et du couvercle pour certains types) et le ravalement des parements. Ainsi, le moindre fragment a priori sans intérêt peut rejoindre pleinement les artéfacts utiles à l'étude d'un site. Pour ne prendre qu'un exemple, l'attention avec laquelle l'abbé André Merlange a noté et dessiné dans ses carnets de terrain le moindre indice archéologique lors de la construction en 1968 de la résidence de la Tournelle à Auxerre (89), à l'emplacement de l'église Saint-Martin-lèsSaint-Marien, mentionnée dès la fin $\mathrm{du} \mathrm{VI}^{\mathrm{e}}$ siècle dans le règlement liturgique de l'évêque Aunaire, confère aujourd'hui encore à ces archives un intérêt particulièrement grand. Il a, entre autres, dessiné les fragments sculptés (éléments funéraires et d'architecture) et ce qui lui semblait être à juste titre des morceaux de sarcophages. Quelques-uns d'entre eux renvoient assurément au Groupe A (dit par ailleurs «à bandes de stries gravées d'obliquité alterné »), produit dans les carrières du sud de l'Auxerrois (vallées de l'Yonne et de la Cure) dès le dernier tiers du Ve siècle (fig. 6) ; ainsi, ces fragments isolés, que d'autres auraient ignorés, participent à la connaissance du site funéraire et religieux, à son positionnement chronologique et deviennent des arguments très précieux.

Fig. 6.
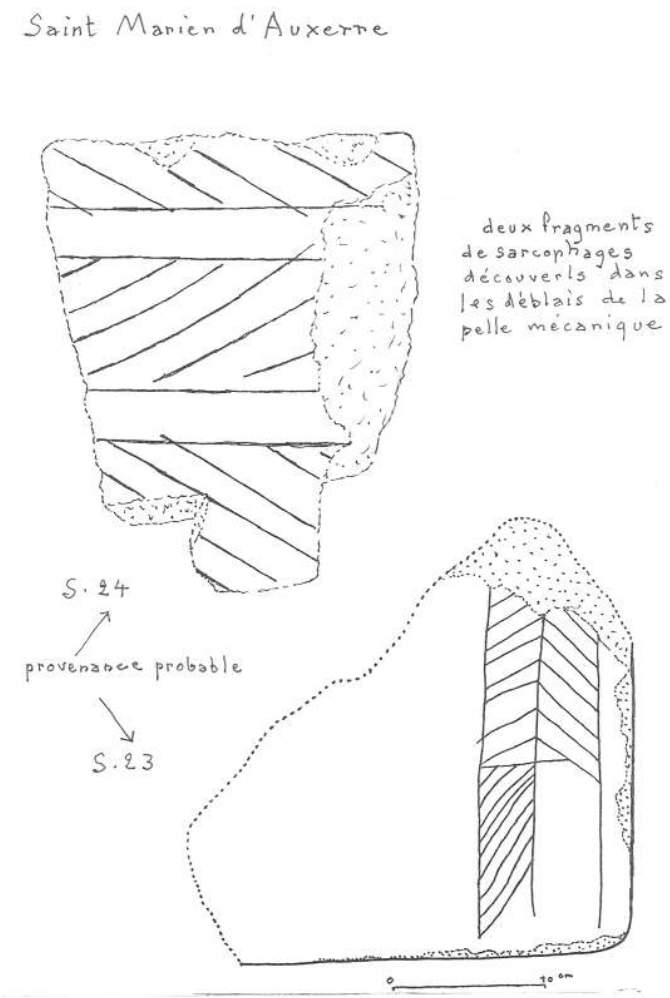

Croquis de fragments de sarcophages (Groupe A), carnet de terrain de l'abbé André Merlange, 1968, conservées au centre d'études médiévales d'Auxerre (Yonne), Saint-Martin-lès-Saint-Marien.

(C) A. Merlange, 1968, archives privées

Dans d'autres cas, c'est la présence de brochage, associé ou non à des traces de polka, qui distingue le fragment de sarcophage d'un simple moellon, ou encore la 
reconnaissance d'altération de la pierre par les liquides de décomposition du cadavre (fig. 7).

Fig. 7.

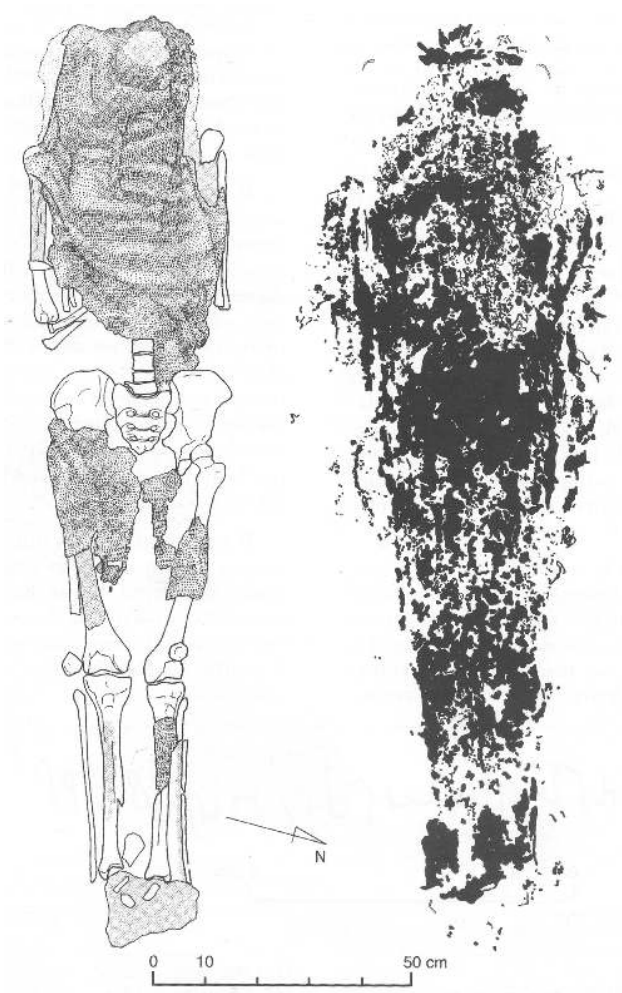

Relevé de l'individu et des traces qu'il a laissées sur le fond de la cuve du sarcophage S. 070, Auxerre (Yonne), abbaye Saint-Germain.

(c) dessin C. Castillo, 1990

Le remploi dans les maçonneries doit également être considéré, phénomène qui a intéressé les chercheurs depuis au moins le XIX ${ }^{\mathrm{e}}$ siècle ${ }^{9}$ et encore aujourd'hui ${ }^{10}$. Ces fragments sont repérés généralement, comme nous l'avons défini, par leur matériau pouvant être différent du reste de la construction, par leur épaisseur standardisée et plus facilement encore lorsque décor ou traces d'outils sont présentés en parement. Les cas sont nombreux et nous ne citerons que ceux de l'église de Cussey-les-Forges (Côted'Or), où les chaînages d'angle du clocher sont constitués de cuves remplies de moellons noyés dans du mortier, le cas de la crypte de l'abbaye Saint-Séverin de Château-Landon (Seine-et-Marne) (fig. 8) ou celui de l'église de Saint-Germain-lèsSenailly, également en Côte-d'Or. Si les contreforts de la nef, largement reprise au XVIII ${ }^{e}$ siècle, comportent de nombreux remplois de fragments de sarcophages, principalement des morceaux de cuves utilisés en carreaux ou en boutisse, c'est le clocher du milieu du XII ${ }^{e}$ siècle qui en contient le plus, masqués toutefois par l'enduit couvrant les maçonneries de moellons. L'église est mentionnée deux fois au IX ${ }^{\mathrm{e}}$ siècle $^{11}$, mais l'archéologie a montré la présence d'une nécropole dès le $\mathrm{VI}^{\mathrm{e}}$ siècle organisée autour d'une tombe de chef à l'ouest et d'un probable mausolée à l'est. Les sarcophages régulièrement mis au jour depuis les premières fouilles de $1910^{12}$ jusqu'aux derniers travaux du début des années $2000^{13}$ appartiennent strictement au type dit 
«bourguignon-champenois », Groupe C de notre typologie, tout comme les fragments remployés depuis au moins le XII ${ }^{\mathrm{e}}$ siècle.

Fig. 8.

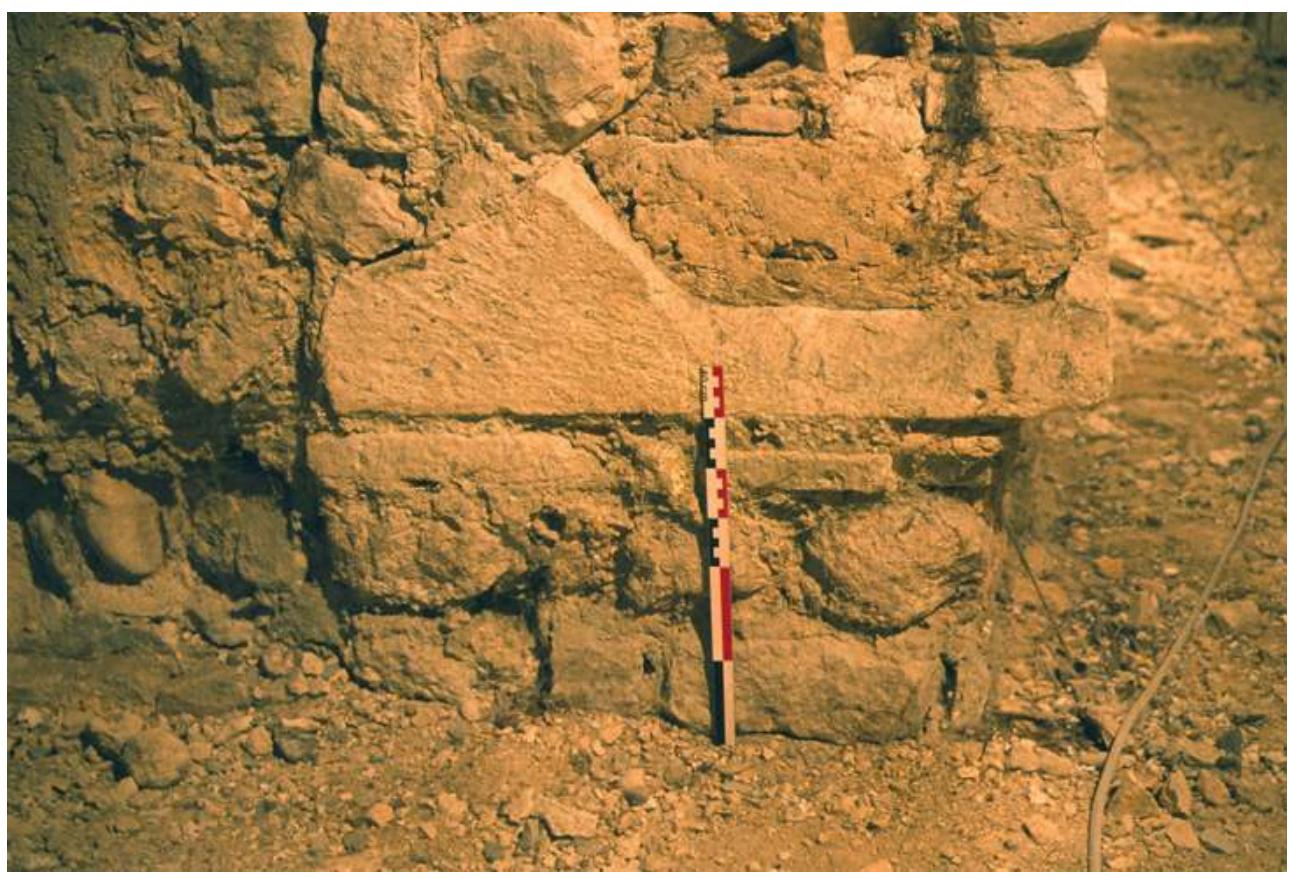

Fragment de cuve remployé dans la construction de la crypte, cuve de sarcophage remployé, Château-Landon (Seine-et-Marne), abbaye Saint-Séverin.

(c) F. Henrion

Le remploi d'éléments de sarcophages peut sans doute renvoyer à la volonté de maintenir dans la construction même de l'édifice communautaire le souvenir à la fois des origines et des défunts ${ }^{14}$. Le maintien dans un nouveau monument des matériaux issus de tombes considérées lors de leur découverte par les constructeurs comme renvoyant aux origines assure, jusque dans les murs, un lien intime entre un passé et un présent. Le remploi de sarcophages dans la construction d'une église peut être considéré comme une volonté ne relevant pas uniquement d'une recherche d'économie. La réutilisation de ces fragments fragilise considérablement les angles et les structures chaînées alors que les bâtisseurs maîtrisent par ailleurs les techniques permettant la mise en œuvre d'un moyen appareil régulier beaucoup plus fiable. La présence dans les murs de pierres identifiables appartenant à des états antérieurs renvoie aux origines mêmes de la communauté, quelle qu'elle soit.

\section{Graffiti, décors fugaces ou accidents}

Il ne s'agit pas ici de minimiser l'importance de l'étude des décors, mais plutôt de montrer la nécessité de lui associer l'étude des autres caractères discriminants; les travaux d'Anne Flammin, dans cette publication, l'illustrent parfaitement. À côté de ceux-ci, il convient de ne pas exclure les interventions secondaires, entre la taille du sarcophage et l'instant de l'inhumation. 

d'un individu mort et le garder en terre. Cette opposition, brièveté du regard des vivants/pérennité de la pierre, est renforcée lorsqu'il y a présence d'un décor plus ou moins élaboré (géométrique ou symbolique) ou d'un travail de taille de qualité et bien maîtrisé dont le résultat final s'apparente à un décor.

21 ou dans la cuve n'est finalement pas si rare. Si la plupart peuvent paraître clairs à la lecture, au moins dans la reconnaissance et le déchiffrage, sinon dans l'interprétation, d'autres réduits à de simples traits gravés sans application apparente, quelle que soit la forme géométrique qu'ils présentent, sont plus délicats à prendre en compte. Il s'agit parfois de simples incisions, pouvant paraitre accidentelles, ou du moins ne pas relever d'une volonté de transmettre un message. Lors de la mise en place d'un sarcophage dans sa fosse, par exemple, l'opération de descente de la cuve peut s'avérer délicate, et le frottement des parois contre celles de la fosse inscrit à jamais dans la matière cet incident anecdotique; et le hasard des dessins ainsi subitement créés risque d'égarer l'observateur. Le couvercle d'un sarcophage de Ligny-le-Châtel (Yonne) ${ }^{15}$ présente en parement des gravures linéaires qui pourraient être considérées comme un acte volontaire (fig. 9). Or, la position de ce sarcophage dans la nécropole, plus haut que les autres en altitude, suggère plutôt des accidents, sans doute consécutifs aux passages répétés de la charrue lors des labours.

Fig. 9.

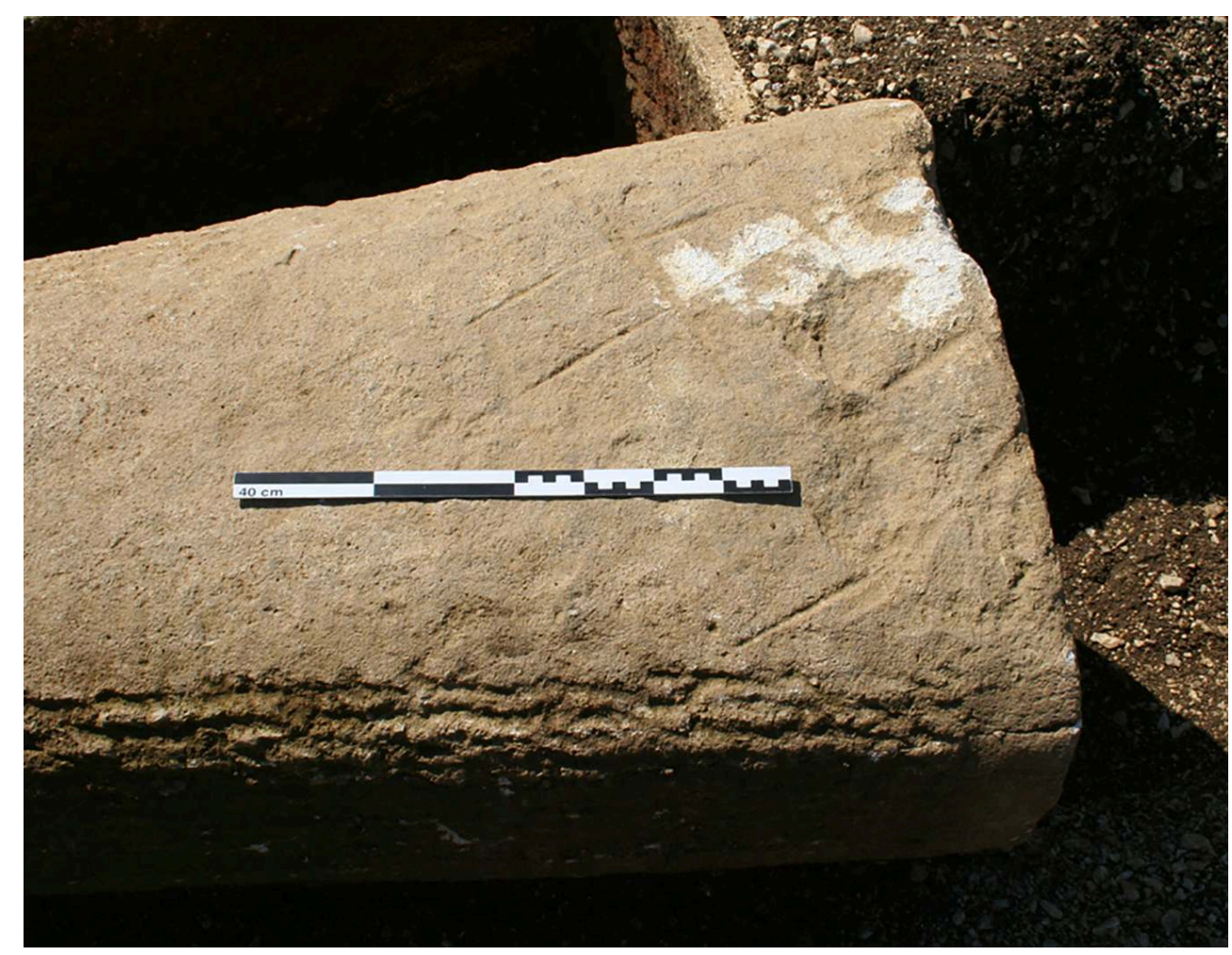

Gravures accidentelles sur le couvercle du sarcophage S. 001, La Coupe au Meunier, Ligny-le-Châtel (Yonne).

(c) F. Henrion 
Les graffiti volontaires sont toutefois nombreux et leur reconnaissance s'inscrit parfaitement dans l'étude de l'objet. Nous citerons seulement pour illustration de ce propos le cas de Bierry-les-Belles-Fontaines (Yonne) avec une francisque (ou ascia?) gravée sur le panneau de tête d'un sarcophage du Groupe $B$, après qu'un méplat a été préparé à la polka (fig. 10), dans la carrière ou sur le lieu même de l'inhumation.

Fig. 10.

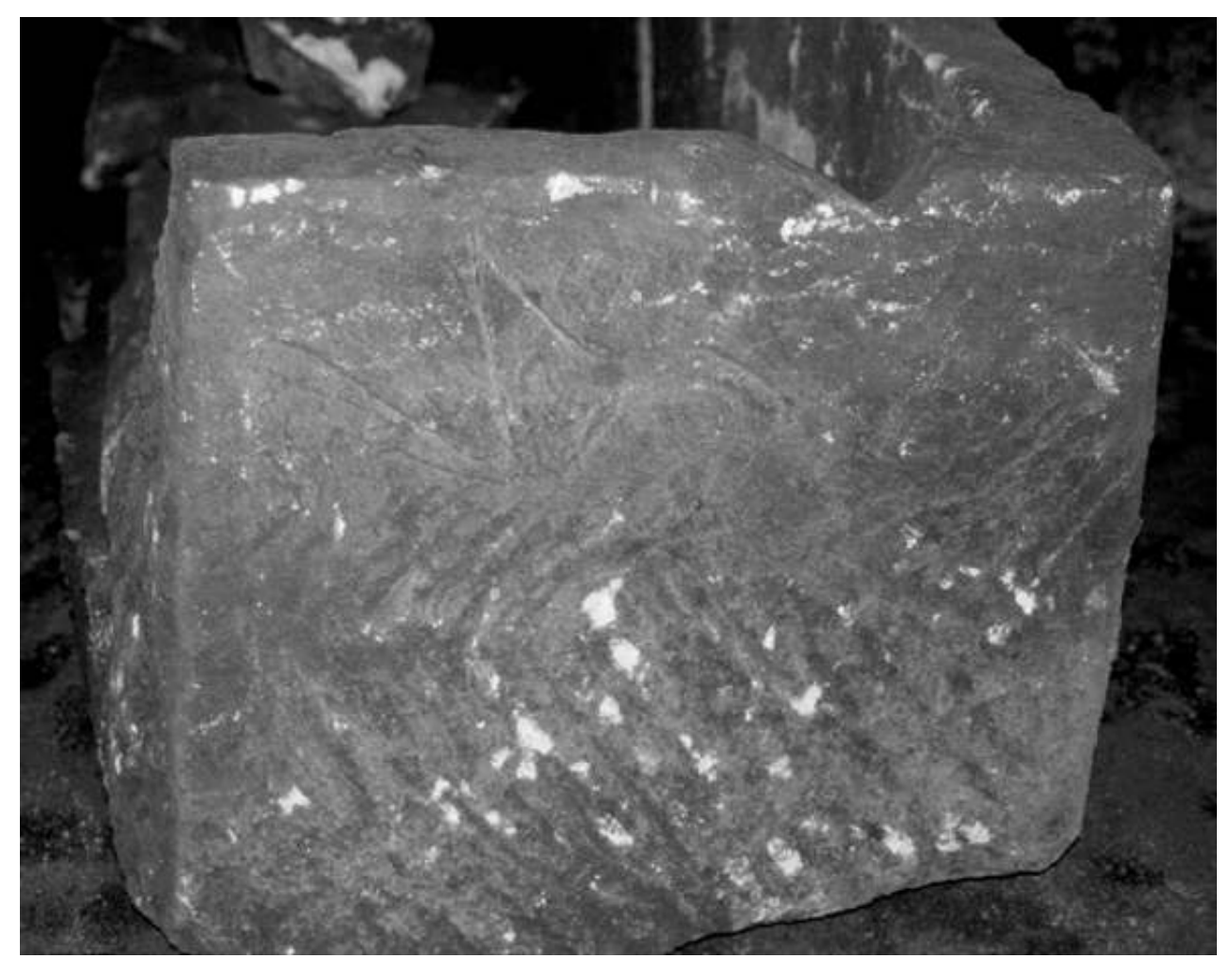

Graffiti sur le panneau de tête du sarcophage S. 156, rue aux Sœurs, Bierry-les-Belles-Fontaines (Yonne).

(c) F. Henrion

23 On connaît des endotaphes pour le Moyen Âge, comme ces ustensiles gravés ou ces plaques de plomb déposées dans la tombe aux côtés du cadavre, mais l'archéologie nous montre également la présence de graffiti sous le couvercle ou dans la cuve pour des sarcophages mérovingiens ou carolingiens : à l'église Saint-Clair de Hérouville-SaintClair (Calvados), avec un bateau gravé dans la cuve, ou encore les deux exemples de Saint-Germain d'Auxerre (Yonne), avec le sarcophage 058 qui présente sur les parois intérieures de la cuve de très nombreux graffiti symboliques (dents de loup, symboles solaires) et une inscription $R[$ o]gabo corda meor(um), c'est-à-dire « je prierai les âmes des miens » (trad. Guiseppe de Spirito), et le sarcophage 070 avec sur le contreparement du couvercle l'inscription ego +ulus dignus discip(ulus), «moi digne disciple ... ». Ces graffiti n'ont pu être tracés qu'au moment de l'inhumation, avant la fermeture du couvercle et, pour le sarcophage 058 , ils n'ont pu être réalisés qu'une fois le défunt déposé dans la cuve comme en témoigne l'étagement de leur emprise ou le sens de l'inscription montrant que le scribe était positionné la tête en bas (fig. 11). 
Fig. 11.

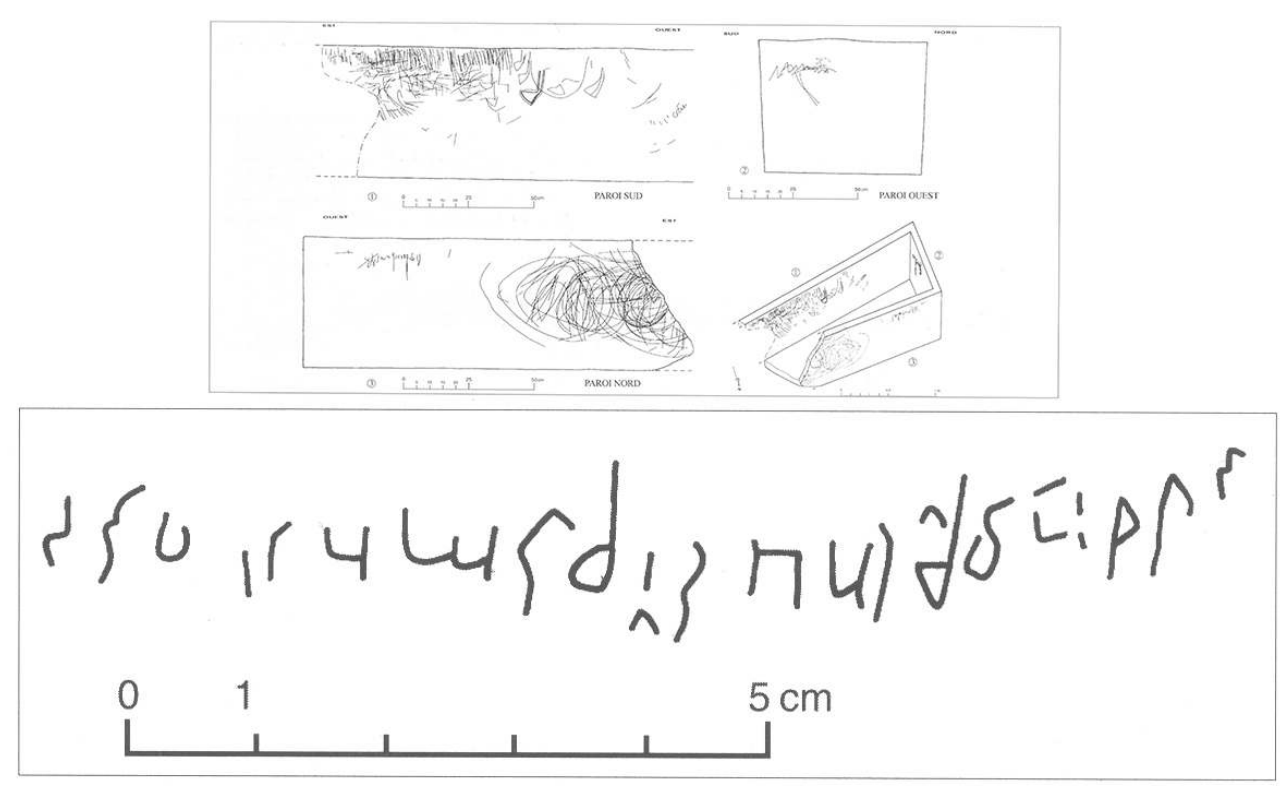

a.Relevés des graffiti dans la cuve du sarcophage S. 058, Auxerre (Yonne), abbaye Saint-Germain. b. Relevé du graffiti sous le couvercle du sarcophage S. 070, Auxerre (Yonne), abbaye Saint-Germain. (c) dessins G. Fèvre

Certaines cuves ou couvercles présentent un décor à peine ébauché, réglé ou gravé à main levée, que parfois seule une lumière rasante permet de déceler. C'est le cas par exemple du sarcophage ML07 conservé dans le musée lapidaire de Saint-Ursanne (Suisse, canton du Jura), avec sur les parois de la cuve un trait vertical délimitant un bandeau à la tête et au pied, et un quadrillage oblique (fig. 12). On ne peut exclure qu'il s'agisse du tracé préparatoire d'un décor plus élaboré, pour des gravures plus profondes dont l'exécution a finalement été abandonnée. 
Fig. 12.

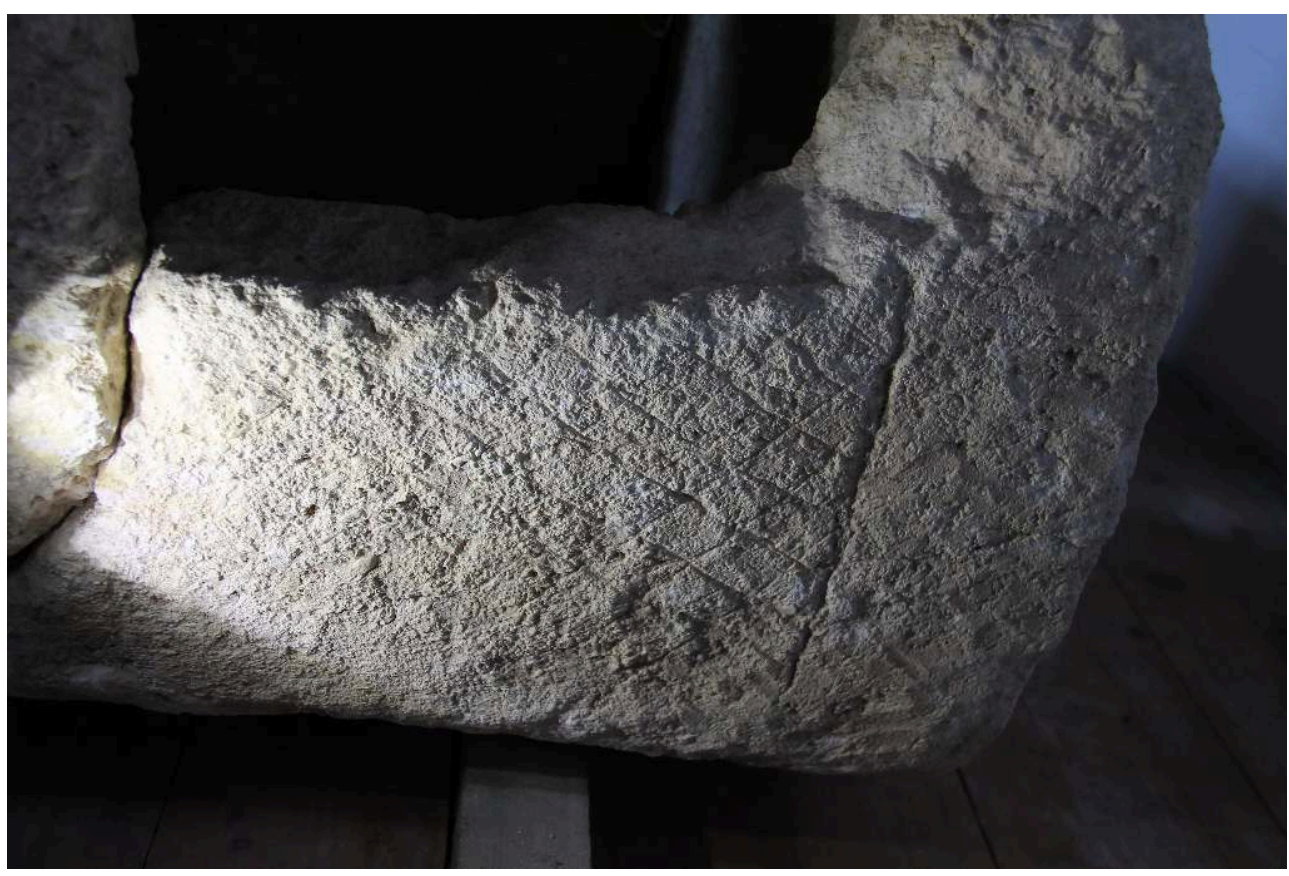

Détail en lumière rasante du décor gravé sur le panneau gauche de la cuve du sarcophage ML07, Saint-Ursanne (Suisse, canton du Jura).

\section{(c) F. Henrion}

Nous avons souhaité, par cette courte note, insister sur la nécessaire prise en compte de l'ensemble des caractères d'un sarcophage de pierre afin d'en comprendre l'histoire et ainsi l'intégrer au mieux à l'étude d'un corpus ou d'un site. Il ne s'agit plus aujourd'hui de ne considérer que les exemplaires décorés, on l'aura compris, et la connaissance contenue dans un simple fragment n'est plus à démontrer. Chaque objet, qu'il soit entier ou fragmentaire, doit être vu comme le marqueur d'une tradition funéraire dans ses dimensions sociale, technique et économique.

\section{NOTES}

1. Léon Coutil, «L'art mérovingien et carolingien », Bulletin et Mémoires de la Société archéologique de Bordeaux, t. 44, 1927, pp. 1-138.

2. Edmond Le Blant, Les sarcophages chrétiens de la Gaule, Paris, Imprimerie nationale, 1886.

3. Jean-François Baratin, «Les sarcophages ornés ou non du Loiret, origine des matériaux », dans $98^{e}$ Congrès national des Sociétés savantes, Section d'archéologie, Saint-Etienne, 1973, pp. 181-190.

4. Pierre Poulain, « L'extraction et la taille des sarcophages dans la carrière de "La Roche Taillée" à Arcy-sur-Cure (Yonne) », Revue archéologique de l'Est et du Centre-Est, t. V, fasc. 1, 1954, pp. 29-45; Michel Cousin, Archéologie des carrières souterraines de Doué-la-Fontaine. À la recherche d'un passé souterrain en Anjou, ARDA, Angers, 2002, 109 p.

5. Voir article dans la présente publication. 
6. Les sarcophages de l'Antiquité tardive et du haut Moyen Âge: fabrication, utilisation, diffusion, actes des $\mathrm{XXX}^{\text {es }}$ journées internationales d'Archéologie Mérovingienne, Bordeaux 2, 3 et 4 octobre 2009, Aquitania, Supplément 34, 2015.

7. Jacques Roger, Les sarcophages du département de la Creuse. Une contribution à l'étude des pratiques funéraires $d u$ haut Moyen Âge, Guéret, Société des sciences naturelles, archéologiques et historiques de la Creuse, 2015 ; Sophie Liegard, Les sarcophages médiévaux du département de l'Allier. Étude des contenants funéraires en pierre des premiers siècles du Moyen Âge dans le Bourbonnais et ses environs, Publication du GRAHCA, 10, Le Montet, 2017.

8. Fabrice Henrion, «La morphologie des sarcophages du haut Moyen Âge comme critère typologique ", dans Archéologie des pratiques funéraires. Approches critiques, Actes de la table ronde des 7 et 9 juin 2001, Bibracte, Centre archéologique européen, 2004, pp. 255-259 et «Étude des sarcophages et méthodologie: l'indispensable croisement des données technologiques, morphologiques et pétrographiques ", dans Les sarcophages de l'Antiquité tardive et du haut Moyen Âge: fabrication, utilisation, diffusion, actes des $\mathrm{XXX}^{\mathrm{es}}$ journées internationales d'Archéologie Mérovingienne, Bordeaux 2, 3 et 4 octobre 2009, Aquitania, Supplément 34, 2015, pp. 33-43.

9. Paul Gauchery, « De l'emploi des cercueils de pierre dans la construction des églises à l'époque romane ", Mémoires de la Société des antiquaires du Centre, XXII, 1897-1898, pp. 81-86.

10. On citera en particulier les travaux de Jean-Pierre Gély et ceux de Sophie Liegard.

11. Vers 840 : In pago Tornodorensi, Quinciacus villa (...), cum duabus ecclesiis, quarum una in honore sancti Martini, altera in venerationne sancti Germani (...), Bibliothèque de l'École des chartes, t. I, 1840, pp 208-209, puis en 858: In pago Tornodorensi, in villa quae vocatur Quintiacus, abbatiolum sancti Germani, Gallia christiana, IV, Instrumenta, 1728, col. 52. La mention datée vers 840 nous apprend que Saint-Germain-lès-Senailly est alors sur le territoire de la villa de Quincy (Quincy-le-Vicomte, commune située à environ $2 \mathrm{~km}$ de Saint-Germain) dont le vocable de l'église paroissiale est encore actuellement Saint-Martin. Le terme abbatiolum de la mention de 858 renvoie assurément à la présence d'une communauté, sans doute modeste d'où l'emploi du diminutif, mais toutefois autonome et dirigée par un abbé. On ne rencontrera plus ce terme, qui peut représenter l'ultime affirmation d'une fonction affaiblie qui disparaîtra ensuite.

12. Anonyme, "La nécropole barbare de Saint-Germain-lès-Senailly ", dans Bulletin de la Société archéologique et biographique de Montbard, 2, 1910, p. 18 et "Nos fouilles à Saint-Germain-lèsSenailly », Bulletin de la Société archéologique et biographique de Montbard, 4, 1911, pp. 27-28.

13. Fouilles préventives sous notre direction, inédites.

14. F. Henrion, «Remplois de sarcophages du haut Moyen Âge et souvenir de leur image à l'époque romane en Bourgogne et alentours ", Les Cahiers de Saint-Michel de Cuxa, XLII, Mémoires, tombeaux et sépultures à l'époque romane, 2011, pp. 93-102.

15. Opération préventive dirigée par Patrick Chopelain - INRAP, 2006.

\section{RÉSUMÉS}

Au cours des deux dernières décennies, l'étude des sarcophages de pierre du haut Moyen Âge a pris un tournant particulier en ne considérant plus «l'objet» seulement pour son décor mais bien pour lui-même, révélant ainsi des caractères discriminants jusqu'alors trop souvent laissés de côté. Ainsi, en tenant compte de l'ensemble de son histoire, depuis la carrière d'où il provient jusqu'à la nécropole, au cimetière ou au lieu de culte qui finalement l'accueille, il est dorénavant 
possible d'inscrire n'importe quel exemplaire, entier ou fragmentaire, dans une typologie et une chronologie archéologiquement établies. Si le décor avait dû être mis de côté dans l'élaboration de la méthode de classification typologique et chronologique, on y revient naturellement, par le prisme des études antérieures et l'apport des données nouvelles.

Over the last two decades, the study of Early Middle Ages stone sarcophagi has taken a particular turn: the "object" itself is now worthy of consideration, not only its decoration, thus revealing the distinguishing characteristics that were often that were previously too often left aside. Thus, by considering the whole of its history, from its quarry of origin to the necropolis, cemetery or place of worship that finally received it, it is now possible to place any specimen, whole or fragmentary, within an archaeologically established typology and chronology. While the decoration had to be put aside during the elaboration of the typological and chronological classification method, we naturally returned to it, through the prism of previous studies and the contribution of new data.

INDEX

Mots-clés : sarcophage de pierre, techniques de mise en œuvre, typologie, fragments, décors gravés, graffiti.

Keywords : stone sarcophagus, techniques, typology, fragments, engraved decoration, graffiti

\section{AUTEUR}

\section{FABRICE HENRION}

Élève diplômé de l'École des hautes études en sciences sociales et titulaire d'un DEA d'archéologie des périodes historiques de l'université de Paris 1 Panthéon-Sorbonne, ses travaux portent sur la production et la diffusion des sarcophages de pierre au haut Moyen Âge et au début du Moyen Âge avec l'étude de grands ensembles ou d'exemplaires isolés, et sur l'archéologie des édifices religieux. Il a notamment dirigé l'étude archéologique de l'ancien prieuré Saint-Sauveur de Melun (Seine-et-Marne), de l'ancien prieuré Notre-Dame de La Charité-sur-Loire (Nièvre) ou encore de l'abbaye Saint-Michel de Cuxa (Pyrénées-Orientales), et participé à aux chantiers archéologiques de Cluny (Saône-et-Loire), Saint-Quentin (Aisne), Vézelay (Yonne), etc. Longtemps responsable d'opérations au Centre d'études médiévales d'Auxerre, il est aujourd'hui conservateur régional adjoint de l'archéologie (Direction régionale des affaires culturelles de Normandie).

Fabrice Henrion is a medieval archaeologist associated with UMR 6298 ARTEHIS Dijon. A graduate of the École des Hautes Études en Sciences Sociales and holder of an advanced degree in the archaeology of historical periods from the Université de Paris 1 Panthéon-Sorbonne, his work focuses on the production and distribution of early medieval sarcophagi with the study of large ensembles or isolated examples, and on the archaeology of religious edifices. He has overseen the archaeological studies of the former Prieuré Saint-Sauveur de Melun, Seine-et-Marne; the former Prieuré Notre-Dame de La Charité-sur-Loire, Nièvre; and the Abbaye Saint-Michel de Cuxa, Pyrénées-Orientales, and has taken part in archaeological projects, including Cluny, Saône-etLoire; Saint-Quentin, Aisne; and Vézelay, Yonne. Long in charge of operations at the Centre d'Études Médiévales d'Auxerre, he is now assistant regional curator of archaeology (Direction Régionale des Affaires Culturelles de Normandie). 